\title{
DERIVATIONS OF COMMUTATIVE BANACH ALGEBRAS
}

\author{
BY PHILIP C. CURTIS, JR. ${ }^{1}$
}

Communicated by John W. Green, January 11, 1961

In [2] Singer and Wermer showed that a bounded derivation in a commutative Banach algebra $\mathfrak{A}$ necessarily maps $\mathfrak{A}$ into the radical $\Re$. They conjectured at this time that the assumption of boundedness could be dropped. It is a corollary of results proved below that if $\mathfrak{A}$ is in addition regular and semi-simple, this is indeed the case.

What is actually proved here is that under the above hypotheses, if $D$ is a derivation of $\mathfrak{A}$ into $C\left(\Phi_{\mathfrak{I}}\right),{ }^{2} \Phi_{\mathfrak{I}}$ the structure space of $\mathfrak{A}$, then $D$ is a bounded operator from $\mathfrak{A}$ to $C\left(\Phi_{\mathfrak{A}}\right)$. The topologies are the norm topology in $\mathfrak{A}$ and the sup norm topology in $C\left(\Phi_{\mathfrak{q}}\right)$. An application of the closed graph theorem shows that if $D$ maps $\mathfrak{A}$ into itself, $D$ must be a bounded operator in $\mathfrak{A}$, hence by the Singer, Wermer theorem, $D=0$.

If $\mathfrak{A}$ is regular but not semi-simple, then it follows from the above that $D$ will map $\mathfrak{Q}$ into $\Re$ provided that $D$ maps $\Re$ into $\Re$. This the author can verify only if $\Re$ is nilpotent.

In what follows $\mathfrak{A}$ will always denote a regular, commutative, semisimple Banach algebra with norm $\|\cdot\|$. Applying the Gelfand isomorphism we will identify $\mathfrak{A}$ and the corresponding subalgebra of $C\left(\Phi_{\mathfrak{q}}\right)$. For convenience we also will assume $\mathfrak{A}$ possesses an identity. It is easily seen that this doesn't affect the generality of the results.

Let $\mathfrak{M}_{\phi}$ be a maximal ideal of $\mathfrak{A}$, and $\phi$ the corresponding point in $\Phi_{\mathfrak{q}}$. It is noted in [2] that there exists a derivation $D$ of $\mathfrak{A}$ into some semi-simple extension $\mathfrak{B}$ of $\mathfrak{A}$ iff $\mathfrak{M}_{\phi}^{2} \neq \mathfrak{M}_{\phi}$ for some maximal ideal $\mathfrak{M}_{\phi}$. In fact $\mathfrak{B}$ may be taken to be $B\left(\Phi_{\mathfrak{Q}}\right)$, the ring of bounded complex functions on $\Phi_{\mathscr{Q}}$. For if this condition is satisfied, following Singer and Wermer, we define by Zorn's Lemma a nontrivial linear functional $f_{\phi}$ on $\mathfrak{A}$ which annihilates $\mathfrak{M}_{\phi}^{2}$ and the identity. If we define $D$ by

$$
\begin{aligned}
& D x\left(\phi^{\prime}\right)=0, \quad \phi^{\prime} \in \Phi_{\mathfrak{A}}, \quad \phi^{\prime} \neq \phi, \quad x \in \mathfrak{A}, \\
& D x(\phi)=f_{\phi}(x),
\end{aligned}
$$

it is easily seen that $D$ is a derivation of $\mathfrak{Y}$ into $B\left(\Phi_{\mathfrak{Y}}\right) . D$ is in general unbounded, but if $\overline{\mathfrak{M}_{\phi}^{2}} \neq \mathfrak{M}_{\phi}, f_{\phi}$, and consequently $D$, may be chosen (via the Hahn-Banach Theorem) to be bounded. Modifying the

${ }^{1}$ This research was supported by the United States Air Force, Office of Scientific Research, under contract AF49(638)-859.

${ }^{2} C\left(\Phi_{\mathscr{Y}}\right)$ denotes the algebra of continuous complex functions on the space $\Phi_{\mathfrak{2}}$. 
terminology of Singer and Wermer somewhat we refer to both the functionals $f_{\phi}$ and the associated operators $D$ as point derivations. The main result of this note is that any derivation $D$ of $\mathfrak{A}$ into $B\left(\Phi_{\mathfrak{x}}\right)$ is the sum of a bounded derivation and finitely many unbounded point derivations.

The key to the argument is the following result from $[1, \S 3]$ stated in a form suitable to our needs.

THEOREM 1. Let $\|\cdot\|_{1}$ be a norm on $\mathfrak{A}$ under which $\mathfrak{A}$ is a normed algebra. Let $\mathrm{G}$ be the class of open sets $G$ for which there exist constants $M_{G}$ satisfying

$$
\|x\|_{1} \leqq M_{G}\|x\|, \quad x \in \mathfrak{A} ; \quad c(x) \subset G .^{3}
$$

Then there exists a finite subset $F$ of $\Phi_{\mathfrak{A}}$, called the singularity set of the norm $\|\cdot\|_{1}$, with the following two properties:

(1) If $G$ is open and $\bar{G} \cap F=\varnothing$, then $G \in g$.

(2) If $G \in \mathcal{G}$, then $G \cap F=\varnothing$.

We now state and prove the result of the note.

TheOREM 2. Let $D$ be a derivation of $\mathfrak{A}$ into $B\left(\Phi_{\mathfrak{x}}\right)$. Then there exists a finite subset $F$ of $\Phi_{\mathfrak{A}}$ and a bounded derivation $D_{1}$ of $\mathfrak{A}$ into $B\left(\Phi_{\mathfrak{X}}\right)$ such that if $D_{2}=D-D_{1}$, then $D_{2} x(\phi)=0, x \in \mathfrak{I}$ and $\phi \in \Phi_{\mathfrak{A}}-F$. For $\phi \in F$, $f_{\phi}(x) \equiv D_{2} x(\phi)$ is an unbounded point derivation. If for each $x \in \mathfrak{A}$, $D x \in C\left(\Phi_{\mathfrak{x}}\right)$, then $F=\varnothing$ and $D$ is a bounded operator.

Proof. Re-norm $\mathfrak{A}$ by defining for $x \in \mathfrak{A}\|x\|_{1}=\|x\|+\|D x\|_{\infty}$ where $\|y\|_{\infty}=\sup _{\phi \in \Phi_{\mathfrak{\eta}}}|y(\phi)|$. Clearly $\mathfrak{A}$ is a normed algebra under $\|\cdot\|_{1}$. Therefore if $F$ is the singularity set for $\|\cdot\|_{1}$, we assert $f_{\phi}(x) \equiv D x(\phi)$ is a bounded linear functional on $\mathfrak{A}$ iff $\phi \notin F$. If $\phi \notin F$, then by the regularity of $\mathfrak{A}$ there exists $h_{\phi} \in \mathfrak{A}$ and a neighborhood $V$ of $F$ such that $h_{\phi}(\phi)=1, h_{\phi}(V)=0$. Let $\Im_{V}=\{x \in \mathfrak{R}: x(V)=0\}$. Choose an open set $W, \bar{W} \cap F=\varnothing$ such that if $x \in \Im_{V}$, then $c(x) \subset W$. Then by Theorem $1, D$ is bounded on $\Im_{r}$. Hence if $\left\{x_{n}\right\}$ is any sequence in $\mathfrak{A}$ tending to zero, then $x_{n} h_{\phi} \in \Im_{V}$ and $x_{n} h_{\phi} \rightarrow 0$. Consequently $D\left(x_{n} h_{\phi}\right) \rightarrow 0$. But $D\left(x_{n} h_{\phi}\right)(\phi)=D x_{n}(\phi)+x_{n}(\phi) \cdot D h_{\phi}(\phi)$. Therefore $f_{\phi}\left(x_{n}\right) \equiv D x_{n}(\phi) \rightarrow 0$. For the converse let $H=\left\{\phi: f_{\phi}\right.$ is bounded on $\left.\mathfrak{A}\right\}$. Since $\|D x\|_{\infty}<\infty$ for each $x \in \mathfrak{A}$, there exists by the principle of uniform boundedness, a constant $M$ such that $\sup _{\phi \in H}|D x(\phi)| \leqq M\|x\|$. If $\phi_{0} \in H \cap F$, pick an open set $G \subset H, \phi_{0} \in G$ and an element $y \in \mathfrak{U}$ for which $y(G)=1$ and $y\left(\Phi_{\mathfrak{A}}-H\right)=0$. Then if $x \in \mathfrak{A}$ and $c(x) \subset G$, we have $x y=x$. Therefore $D x=y D x+x D y$ and

\footnotetext{
$3(x)$ denotes the carrier of the function $x$.
} 


$$
\begin{aligned}
\|D x\|_{\infty} & \leqq \sup _{\phi \in H}|y(\phi) \cdot D x(\phi)|+\|x\| \cdot\|D y\|_{\infty} \\
& \leqq\left\{\|y\|_{\infty} \cdot M+\|D y\|_{\infty}\right\}\|x\| .
\end{aligned}
$$

This contradicts property (2) of Theorem 1.

If $F \neq \varnothing$ and $D$ is unbounded, we define $D_{1}$ by

$$
\begin{aligned}
D_{1} x(\phi) & =D x(\phi), & & \phi \notin F, \\
& =0, & & \phi \in F .
\end{aligned}
$$

Again applying the uniform boundedness principle it follows that $D_{1}$ is a bounded operator from $\mathfrak{A}$ to $B\left(\Phi_{\mathfrak{A}}\right)$. The statement about $D_{2}$ is clear.

To complete the proof we observe first that if $\phi$ is isolated in $\Phi_{\mathfrak{A}}$, then $\phi \notin F$. In fact for such $\phi, D x(\phi)=0$. For let $k_{\phi}$ be the characteristic function of $\{\phi\}$. Then $k_{\phi} \in \mathfrak{A}$ and for $x \in \mathfrak{U} D\left(k_{\phi} x\right)(\phi)=0$. Hence $D x(\phi)=-x(\phi) \cdot D k_{\phi}(\phi)=0$. Consequently $\overline{\Phi_{\mathscr{Y}}-F}=\Phi_{\mathscr{Q}}$. Therefore if for each $x \in \mathfrak{A}, D x$ is a continuous function on $\Phi_{\mathfrak{A}}$, it follows that $\|D x\|_{\infty}=\sup _{\phi \in \Phi \mathfrak{Q}-F}|D x(\phi)| \leqq M\|x\|$. This completes the proof.

Corollary. Let $\mathfrak{B}$ be a subalgebra of $C\left(\Phi_{\mathfrak{A}}\right)$ containing $\mathfrak{A}$. If $\mathfrak{B}$ is a Banach algebra under some norm and $D$ is a derivation of $\mathfrak{A}$ into $\mathfrak{B}$, then $D$ is a bounded operator. If $D$ maps $\mathfrak{A}$ into itself, then $D \equiv 0$.

Proof. The first result follows by the closed graph theorem. An application of the theorem of Singer and Wermer [2] then yields the second.

If now $\mathfrak{A}$ is not semi-simple and $D$ maps $\mathfrak{A}$ into itself, then one may factor out the radical and apply the above corollary to prove that $D$ maps $\mathfrak{A}$ into $\Re$ provided that $D$ maps $\Re$ into $\Re$. If $\Re$ is nilpotent, this follows. For if $x^{n}=0$, then $0=D^{n} x^{n}=n !(D x)^{n}+$ terms each of which involves a positive power of $x$, hence belongs to the radical. Therefore $(D x)^{n} \in R$, and consequently $D x \in R$.

The validity of this result for non-nilpotent radicals is unknown to the author. Without some topological assumptions the result is of course false. Ordinary differentiation in the ring of formal power series is a derivation which does not map the radical into itself.

\section{BIBLIOGRAPHY}

1. W. G. Bade and P. C. Curtis, Jr., Homomorphisms of commutative Banach algebras, Amer. J. Math. vol. 82 (1960) pp. 589-608.

2. I. M. Singer and J. Wermer, Derivations on commutative normed algebras, Math. Ann. vol. 129 (1955) pp. 260-264.

University of Californta, Los Angeles 